\title{
Reliability and risk controlled by the simultaneous presence of random events on a time interval
}

\author{
M.T.Todinov \\ Department of Mechanical Engineering and Mathematical Sciences \\ Oxford Brookes University, Oxford, OX33 1HX, UK \\ email: mtodinov@brookes.ac.uk
}

\begin{abstract}
The paper treats the important problem related to risk controlled by the simultaneous presence of critical events, randomly appearing on a time interval and shows that the expected time fraction of simultaneously present events is insensitive to the distribution of events durations. In addition the paper shows that the probability of simultaneous presence of critical events is practically insensitive to the distribution of the events durations. These counter-intuitive results provides the powerful opportunity to evaluate the risk of overlapping of random events through the mean duration times of the events only, without requiring the distributions of the events durations, their variance or the mixing proportions of the individual distributions, in the case of duration times represented by distribution mixtures.

A closed-form expression for the expected fraction of unsatisfied demand for random events following a homogeneous Poisson process in a time interval is introduced for the first time. In addition, a closed-form expression related to the expected time fraction of unsatisfied demand, for a fixed number of consumers initiating random demands with a specified probability, is also introduced for the first time.

The concepts stochastic separation of random events based on the probability of overlapping and the average overlapped fraction are also introduced. Methods for providing stochastic separation and optimal stochastic separation achieving balance between risk and cost of risk reduction are presented.
\end{abstract}

Keywords: stochastic separation, random demand, unsatisfied demand, constraint on resources, time interval, probability, risk

\section{Introduction}

Reliability and risk are often controlled by the simultaneous presence of critical events on a time interval. Each critical event is characterised by a particular duration which is a random variable and can appear randomly, at any time during an operational interval with given length. The simultaneous appearance of two or more critical events during the operational interval is a common source of system failures.

The first major category of this type of failure is present when the simultaneous appearance of critical events has engaged servicing resources to the point where a servicing resource is no longer available for a new critical event. The lack of intervention resources for repair often leads to further failures with severe consequences.

Consider for example, the breakdowns of heating elements attached to different sections of a long subsea oil pipeline. Each of the failed heating elements demands the intervention of a special repair vessel. If a single intervention vessel is available and a breakdown of a heating element occurs, the repair vessel will be engaged in the repair of the failed heating element. If, during the repair, another breakdown of a heating element occurs, no free repair vessel will be available to service the new repair. As a result, the delay in conducting the second repair will cause the formation of waxy deposits in the affected pipeline section which will block the flow in the pipeline. Blocking the flow in the pipeline by waxy deposits entails 
lengthy and expensive intervention involving cutting and replacing large sections of the blocked pipeline.

The second major category of failure controlled by the simultaneous presence of critical events is present when the simultaneous appearance of critical events increases the load on the system to a level which exceeds the system's strength. Commonly, the simultaneous appearance of random demands whose number is greater than the capacity of the system leads to overloading, with catastrophic consequences for the system.

The third major category of failures relates to the case where some of the critical events weaken/degrade the system's strength while some of the events increase the load on the system. Thus, the simultaneous presence of the critical event 'repair of a failed power line' and the critical event 'sudden increase in power consumption' could also lead to overloading and failure of other power lines and completely disrupt the power supply.

The forth major category of failures relates to the case where the simultaneous presence of critical events results in system failures while individually, none of the critical events can precipitate failure. A typical example is the clustering of the critical event 'presence of sparks' and the critical event 'presence of flammable gases'. A source of sparks is for example present if, for a certain period of time, an electromotor with brushes is used. Flammable gases could result from accidentally spilled fuel which is an event, also associated with certain duration. Explosion is possible if the two events are simultaneously present (overlap).

The maximal tolerable degree of overlapping of risk-critical random events on a time interval varies significantly. It depends on the magnitude of the consequences resulting from the simultaneous presence of risk-critical events. The maximal tolerable level of overlapping must be set individually by the risk experts in the respective application area.

A very small overlap (simultaneous presence)) of random demands can be tolerated for example, in a situation where critically injured people demand a particular piece of lifesaving equipment. In this case, the consequences of unsatisfied demand can be very serious and the maximum tolerable level of the risk of simultaneous presence of critical demands is very low.

A larger degree of simultaneous presence of risk-critical events can be tolerated in some manufacturing processes where a number of machines demand control equipment, production equipment or an operator, at a random time during the production process.

The discussed examples emphasise the urgent need for new reliability and risk measures based (i) on the probability of a simultaneous presence of critical events appearing randomly on a time interval and (ii) on the expected time fraction during which randomly appearing critical events are simultaneously present on a time interval.

The problem related to estimating the risk of simultaneously present critical events is essentially a problem from geometric probability, where a segment of specified length $L$ is covered by randomly located smaller segments with different lengths. The segment with length $L$ represents the operational interval while the smaller segments represent the durations of the critical random events. Simultaneously present critical events exist if and only if overlapping among randomly located smaller segments is present.

There have been a number of publications related to covering the circumference of a circle with segments or a linear segment with segments [1-8]. However, analytical treatment of key problems related to estimating the expected lineal fraction covered by $m$ or more random segments has been presented only recently [9].

Despite the progress made in this direction, (i) the dependence of the overlapped time fraction on the distribution of the duration times, (ii) the solution of the case related to consumers initiating random demands with a specified probability and (iii) the expected fraction of unsatisfied demand for random events following a Poisson process on a time 
interval are still open questions.

In queuing theory, the Poisson process has been traditionally used as a statistical model for random events occurring in a time interval. Despite the abundance of publications on queuing systems, none of the published classical studies [10-14], nor more recent texts on different problems in queuing theory [15-19] nor recent text on probability [20-22] treats the key question related to the dependence of the probability of overlapping of random events on a time interval on the distributions of their durations.

To the best of the author's knowledge, no theoretical models have ever been reported related to (i) the expected time fraction of an overlap of a particular order for risk-critical random events following a homogeneous Poisson process in a time interval and (ii) the expected time fraction of an overlap for random events initiated with some probability by a given number of consumers.

Furthermore, in queuing theory, a central assumption in deriving the expected lengths of queues is the negative exponential distribution of the waiting (servicing) times. As it will be demonstrated later, the distribution of the servicing times for a system which includes different types of components is not the negative exponential distribution; it is a distribution mixture.

Accordingly, the present paper aims to fill the identified gaps by providing a comprehensive treatment of the outlined key questions.

\section{Insensitivity of the probability of a simultaneous presence of critical events to the distribution of their durations}

The risk measure probability of a simultaneous presence of randomly appearing critical events on a time interval is appropriate in cases where almost no overlapping of critical events can be tolerated on a time interval. The dependence of the probability of simultaneous presence on the distributions of the durations of the random events is a key property of the new risk measure, and it will be investigated by using Monte Carlo simulations.

\subsection{Simulation experiments}

The critical risk-controlling events were selected to be $n$ random demands serviced by $m$ sources $(n>m)$. The simulations were performed by an algorithm counting the number of instances of event overlaps of order $m+1$ or higher. An event overlapping of degree $m+1$ or higher is present if more than $m$ events are simultaneously present on the time interval. Details related to the pseudo-code of the simulation algorithm for determining the overlapped fraction of degree $m+1$ or higher have been omitted.

\subsubsection{Simulation experiments with single distributions}

The first simulation involved demand times following a log-normal distribution with mean $140 \mathrm{~min}$, coming from 36 users over a time interval with length 60000 min (1000 hours). Each of the 36 users initiates exactly one demand, randomly located on the operational time interval $(0,60000 \mathrm{~min})$. Simulation experiments with a single source and two sources were conducted.

The simulation results shown in Fig.1 have been obtained after incrementing the standard deviation of the demand times by a step of $5 \mathrm{~min}$. The calculated probabilities of unsatisfied demand of 0.95 and 0.1 , correspond to one and two sources servicing the demands.

If the probability of unsatisfied demand is calculated with a constant length of random demand equal to the mean of $140 \mathrm{~min}$ of the log-normal distribution, the same values 0.95 and 0.1 are obtained. The results presented in Figure 1 show that the probability of unsatisfied demand is practically insensitive to the variance of the random demands. 
From the results presented in Figure 1, it can also be concluded that the inclusion of an additional source servicing the random demands reduces dramatically the probability of unsatisfied demand (from $95 \%$ to only $10 \%$ in the example from Fig. 1 )

In the next simulation experiment, the log-normal distribution of the demand times has been replaced by a normal distribution with the same mean (140 min). The number of users (36) and the length of the operation interval (1000 hours) were kept the same as in the previous simulation experiment. Simulation experiments with a single source and two sources were performed and the standard deviation of the normal distribution was varied with a step of $5 \mathrm{~min}$. The results were almost identical to the results in Fig.1 (not shown here) and indicate that the probability of unsatisfied demand is practically insensitive to the type of the distribution, provided that the mean of the distributions remains the same. The calculated probabilities of unsatisfied demand were again 0.95 and 0.1 , corresponding to one and two sources. In the next simulation experiment, a uniform distribution of the duration of random demands has been used, with a probability density function $f(t)=\frac{1}{2 a}$ if $140-a \leq t \leq 140+a$ and $f(t)=0$ if $t>140+a$ or $t<140+a$, where $t$ is the time. The parameter $a$ determines the spread of the uniform distribution. The uniform distribution and its parameter have been specified in such a way that its mean $(140 \mathrm{~min})$ coincides with the mean of the log-normal distribution used in the previous simulation. Again, the random demands come from 36 users over a time interval with length 60000 min (1000 hours). Each of the 36 users initiates exactly one demand, randomly located along the operational time interval of 1000 hours. Simulation experiments with a single source and two sources were performed. The results for the probability of unsatisfied demand, for a different spread ' $a$ ' of the distribution, are shown in Fig.2.

As can be verified from Fig.2, the probabilities of unsatisfied demand characterising the uniform distribution are almost identical to the probabilities of unsatisfied demand Fig. 1 characterising the log-normal distribution and the normal distribution.

In the next simulation experiment, a triangular distribution, with probability density function $f(t)=0.004762 \times(1-t / 420)$ has been used as a model of the demand times. The distribution function and the parameters of the triangular distribution have been specified in such a way that its mean (140 min) coincides with the means of the log-normal distribution, the normal distribution and the uniform distribution used in the previous simulations.

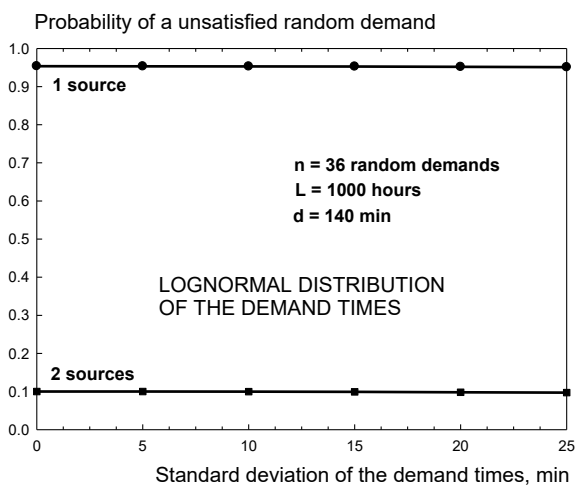

Figure 1. Probability of unsatisfied random demand as a function of the variance of the log-normal distribution for the demand times. 


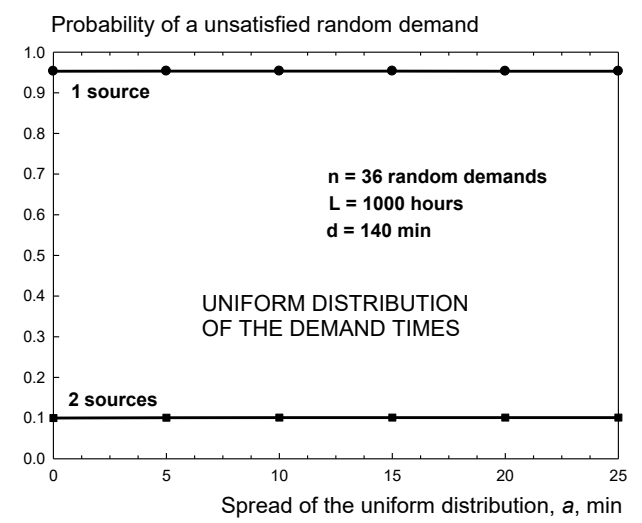

Figure 2. Probability of unsatisfied random demand as a function of the variance of the uniform distribution modelling the demand times.

The result from the simulation, based on demand times following a triangular distribution, were again: 0.95 for the probability of unsatisfied demand if a single source is present and 0.1 if two sources are present.

\subsubsection{Simulation experiments with distribution mixtures}

In general, the distribution of the duration times of random events is neither normal, exponential, lognormal nor any standard distribution. This can be demonstrated immediately, by considering the distribution of the repair times in a computer network composed of $m$ different types of components (e.g. switches, routers, repeaters, servers, cables, printers, etc.). Each component type is characterised by a specific time to repair distribution $F_{i}(t)$ which gives the probability that the time to repair $T$ will be smaller than a specified value $t$ : $F_{i}(t) \equiv \operatorname{Pr}(T \leq t)$. The hazard/failure rate of the type- $i$ component in the network will be denoted by $\lambda_{i}$. The distribution of the repair time given that a failure of a device has occurred then follows a distribution mixture.

Indeed, the repair time $T$ of a failed device can be smaller than $t$ in $m$ distinct mutually exclusive ways. The repair time $T$ can be smaller than $t$ if the device belongs to the first type of components and its time to repair is smaller than $t$. The repair time can also be smaller than $t$ if the device belongs to the second type of components and its time to repair $T$ is smaller than $t$ and so on. The probability $p_{i}$ that given failure, the failed component will be of type $i$ is given by

$$
p_{i}=\lambda_{i} / \sum_{i=1}^{m} \lambda_{i}
$$

where $\lambda_{i}$ is the hazard/failure rate of components from type $i$. The probability that the failed component will be of type $i$ and its repair time will be smaller than $t$ is given by the product $p_{i} F_{i}(t)$, where $p_{i}$ is given by equation (1). Applying the total probability theorem yields the equation

$$
F(t)=p_{1} F_{1}(t)+p_{2} F_{2}(t)+\ldots+p_{m} F_{m}(t)
$$

for the distribution of the time to repair, which is a mixture of distributions.

Consequently, simulation experiments using distributions of demand times modelled by distribution mixtures were conducted. The first simulation experiment involved a single source and 4 users placing random demands. The demand times are coming from a distribution mixture defined as follows. With probability $p_{1}=0.7$, a demand time of 70 minutes is selected and with probability $p_{2}=0.3$, a demand time of 40 minutes is selected. 
The mean $\mu$ of the demand time is therefore: $\mu=p_{1} \mu_{1}+p_{2} \mu_{2}=61$ minutes while the duration of the operation interval was $2880 \mathrm{~min}$ (48 hours).

Two simulations were performed, the first of which had constant demand times of 61 minutes. The second simulation used demand times sampled from the as-defined distribution mixture. Both simulations produced the same result for the probability of unsatisfied demand: 0.23 . The next simulation experiment consisted of reversing the probability of sampling of the distributions. With probability $p_{1}=0.3$, a demand time of duration $\mu_{1}=70$ minutes was selected and with probability $p_{2}=0.7$ a demand time of duration $\mu_{2}=40$ minutes was selected. The mean of the demand time is therefore: $\mu=p_{1} \mu_{1}+p_{2} \mu_{2}=49$ minutes.

The simulation resulted in 0.189 for the probability of unsatisfied demand, which coincided with the result 0.189 obtained for the probability of unsatisfied demand by using constant demand times of duration 49 minutes, equal to the mean of the distribution mixture.

The next simulation experiments involved 8 random demands, a single source and two sources, sequentially. The random demand times were sampled from a distribution mixture composed of two normal distributions with means $\mu_{1}=140 \mathrm{~min}$ and $\mu_{2}=80 \mathrm{~min}$, sampled with probabilities $p_{1}=0.4$ and $p_{2}=0.6$, respectively.

The standard deviations of the normal distributions composing the distribution mixture were increased simultaneously, with a step 4 minutes. The results are shown in Fig.3.

As can be verified from Fig.3, for both a single source and two sources, the probability of unsatisfied random demand is practically insensitive to the variance of the distributions composing the mixture. Including an additional source resulted in a dramatic decrease of the probability of unsatisfied demand, from 0.9 to 0.16 .

The next simulation involved a single source, two sources and only $n=2$ random demands. The random demand times were sampled from a distribution mixture composed of two uniform distributions: $f_{1}(t)=\frac{1}{2 a}$ in the interval $500-a \leq t \leq 500+a$ and $f_{2}(t)=\frac{1}{2 a}$ in the interval $400-a \leq t \leq 400+a$, where $a$ is the half-width of the uniform distributions. The distributions were centred at means $\mu_{1}=500 \mathrm{~min}$ and $\mu_{2}=400 \mathrm{~min}$, and were sampled with probabilities $p_{1}=0.4$ and $p_{2}=0.6$, respectively.

As can be verified from the results in Fig.4, even for the smallest possible number of random demands $(\mathrm{n}=2)$ which could result in unsatisfied demand, the simulated probability of unsatisfied demand (0.28) is still practically insensitive to the variance of the durations of the random demands. A simulated probability of unsatisfied demand equal to 0.28 was obtained by assuming random demands with constant duration of $440 \mathrm{~min}$, equal to the mean of the distribution mixture: $\mu=p_{1} \mu_{1}+p_{2} \mu_{2}=0.6 \times 400+0.4 \times 500=440 \mathrm{~min}$.

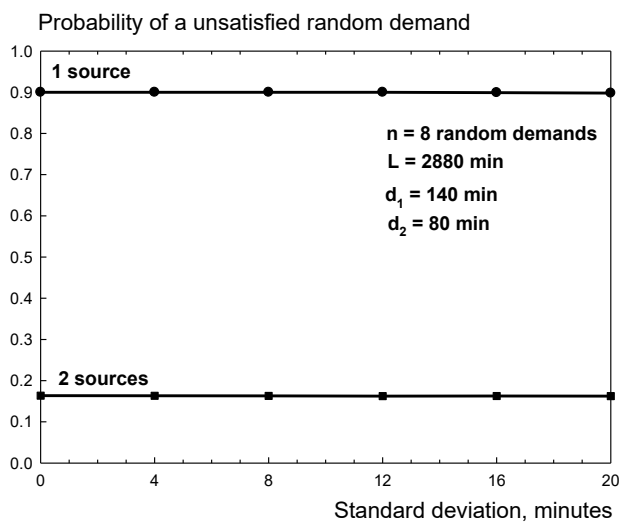


Figure 3. Probability of unsatisfied random demand as a function of the variance of the normal distributions composing the mixture.

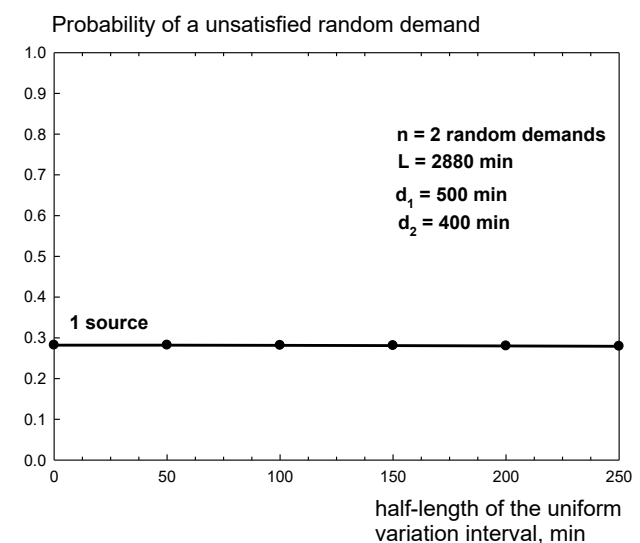

Figure 4. Probability of unsatisfied random demand as a function of the half-length of the rectangular distributions composing the mixture.

The simulations were repeated with normal distributions with means $\mu_{1}=400$ and $\mu_{2}=500$ composing the mixture and standard deviations $20 \mathrm{~min}$, with only 2 random demands arriving on the time interval of $2880 \mathrm{~min}$ (48 hours). The results for the probability of unsatisfied demand were the same (0.28) as in the simulation involving uniform single distributions composing the mixture.

The last set of simulation experiments involved altering the distance between the means of the individual distributions composing the mixture by keeping the grand mean $\mu=440$ of the distribution mixture constant. According to an equation derived in [23], the variance $V$ of a distribution mixture is given by

$$
V=\sum_{i=1}^{m} p_{i}\left[V_{i}+\left(\mu_{i}-\mu\right)^{2}\right], i=1, \ldots, m
$$

where $m$ is the number of individual distributions composing the mixture; $V_{i}$ are the variances of the individual distributions composing the mixture; $p_{i}$ are the probabilities of sampling the individual distributions (the mixing proportions) $\left(\sum_{i=1}^{m} p_{i}=1\right) ; \mu_{i}$ are the means of the individual distributions and $\mu$ is the mean of the distribution mixture. The mean of the distribution mixture is determined from $\mu=\sum_{i=1}^{m} p_{i} \mu_{i}$ [24].

Altering the variance of the distribution mixture by altering the variances of the individual distributions has already been explored in the previous simulations. Consequently, the next simulation experiment involved altering the means $\mu_{1}$ and $\mu_{2}$ of the individual distributions in such a way that the mean $\mu$ of the distribution mixture remains equal to $\mu=440 \mathrm{~min}$.

The first simulation involved means of the individual distributions altered to $\mu_{1}=300$ and $\mu_{2}=650$, correspondingly. As can be verified, this alteration of the means of the individual distributions does not change the mean of the distribution mixture:

$$
\mu=p_{1} \mu_{1}+p_{2} \mu_{2}=0.6 \times 300+0.4 \times 650=440 \mathrm{~min}
$$

The mean of the distribution mixture remains the same $(440 \mathrm{~min})$ as in the simulation involving individual distributions with means $\mu_{1}=400$ and $\mu_{2}=500$. The standard deviations of the individual distributions were kept the same: 20 min. The number of random demands was only 2 , serviced by a single source. 
The simulation with the altered distribution mixture, resulted in a probability of unsatisfied demand equal to 0.28 , the same as the probability obtained in the previous simulation.

The next simulation experiment involved another alteration of the means of the individual distributions to $\mu_{1}=200$ and $\mu_{2}=800$, correspondingly. As can be verified, the altered means do not change the mean of the distribution mixture:

$$
\mu=p_{1} \mu_{1}+p_{2} \mu_{2}=0.6 \times 200+0.4 \times 800=440 \mathrm{~min}
$$

The standard deviations of the individual distributions were kept the same: $20 \mathrm{~min}$. The simulation with the altered distribution mixture, resulted in a probability of unsatisfied demand equal to 0.27 , a value which is very close to the probability estimated from the previous simulations.

These results clearly indicate that the type of the distributions of the critical events controlling the risk and the variance of the duration times of these events practically have no impact on the probability of a simultaneous presence of the events as long as the mean remains the same.

Simulations using duration times sampled from a distribution mixture produce identical results for the probability of a simultaneous presence of events as simulations using duration times equal to the mean of the distribution mixture. Furthermore, even in the case of two randomly appearing events on a time interval, the simulated probability of a simultaneous presence of the events is still practically insensitive to the variance of their durations.

These unexpected results provide the powerful opportunity to estimate the risk of a simultaneous presence of critical events, randomly appearing on a time interval, through the mean duration times only, without requiring the distributions of the duration times, their variance or the mixing proportions of the individual distributions in the common case where the duration times follow a distribution mixture.

\section{Stochastic separation of random demands based on the probability of overlapping}

Consider a finite time interval during which a fixed number of consumers place a demand for a particular service independently and randomly. The durations of the random demands come from a uniform distribution with mean $\mu=140 \mathrm{~min}$, with a range $[\mu-25, \mu+25]$ min. Unsatisfied demand occurs if a random demand arrives while all sources are engaged in servicing other random demands. Suppose that the maximum tolerable probability of unsatisfied demand is $\alpha=20 \%$ and the random demands follow a Homogeneous Poisson process on an operational time interval of 48 hours.

At a specified level of unsatisfied random demand ( $\alpha=20 \%$ ), the maximum frequency of the random demands that can be satisfied by a given number of sources can be determined such that the probability of unsatisfied demand does not exceed a specified tolerable level.

This process will be referred to as stochastic separation of random events based on a specified probability of overlapping. In the stochastic separation of random events based on the probability of overlapping, the absence of overlapping of the random events is guaranteed with a specified probability.

For random demands with number densities ranging from 0.01 hour $^{-1}$ to 0.3 hour $^{-1}$, the simulated probability of unsatisfied demand has been plotted as is shown in Fig.5. During the simulations, the actual number of random demands on the time interval was a random variable whose realisations were obtained by sampling the Poisson distribution.

The curves correspond to a single source, two sources and three sources servicing the random demands. The intersections of the horizontal line corresponding to the maximum acceptable probability of unsatisfied demand with the curves, determine the optimal number densities of random demands equal to 0.05 for a single source, 0.14 for two sources and 0.265 for three sources. Number densities of demands larger than the optimal values lead to 
probability of unsatisfied demand larger than the maximum tolerable value of $20 \%$. Number densities smaller than the optimal values, result in an inefficient use of the available sources and unnecessary restriction of the volume of random demands that can potentially be serviced. The obtained number densities provide not only a stochastic separation of the random events at the maximum tolerable probability of overlapping of $20 \%$; they also guarantee that the specified stochastic separation is characterised by a minimum cost. The optimal stochastic separation of random events is characterised by an optimal balance between the probability of overlapping and the cost for reducing the probability of overlapping.

The results in Fig.5 also confirm the conclusion made earlier, that the inclusion of an extra source reduces drastically the probability of unsatisfied demand. Thus, for a density of random demands equal to 0.14 , the probability of unsatisfied random demand for a single source is 0.77 . The inclusion of a second source reduced the probability of unsatisfied demand to 0.2 .

Consequently, including a second resource drastically improved the degree of stochastic separation of random events.

The results from Fig.5 can also be used to determine the number of sources servicing a specified volume of random demands which provide a degree of stochastic separation (probability of unsatisfied demand) of $20 \%$.

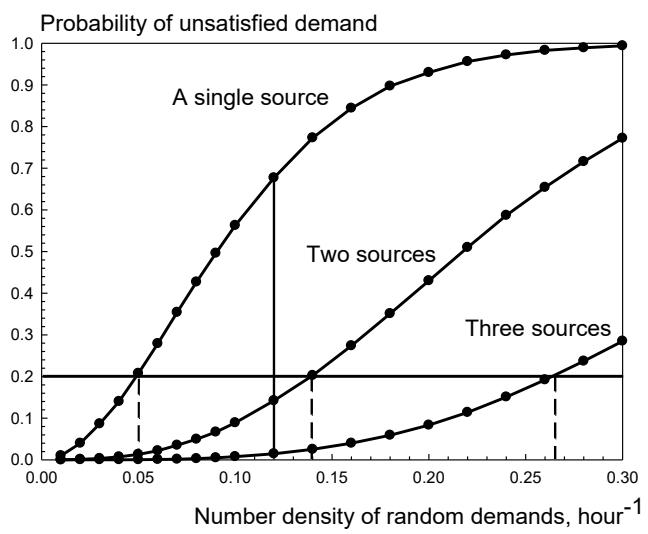

Figure 5. Probability of unsatisfied random demand as a function of the number density of random demands, for a different number of sources.

Thus, for a density of random demands equal to 0.12 hour $^{-1}$, the optimum number of sources is 2 . This value can be determined from the closest intersection to the maximum tolerable probability level of $20 \%$ of the vertical line corresponding to a number density of 0.12 and the curves (Fig.5). A single source leads to an insufficient degree of stochastic separation because the probability of unsatisfied demand is equal to 0.677 . Three sources yield a too low probability of unsatisfied demand equal to 0.014 , which is associated with unnecessary investment in extra sources. The optimal degree of stochastic separation corresponds to a probability of unsatisfied demand ( 0.142$)$ and is obtained from 2 sources.

The proper level of stochastic separation is the key to finding the optimal balance between the number of available sources and the risk of unsatisfied demand. More supplied sources than the optimal number is costly and undermines the profitability of the enterprise; fewer supplied sources than the optimal number increases the risk of unsatisfied demand.

\section{Insensitivity of the expected time fraction of simultaneous presence of critical events to the distribution of their duration times}

The expected time fraction of simultaneous presence of critical events can also be used as a 
powerful measure of the risk of simultaneous presence of random events. Consider the common case where the randomly appearing events are random demands for a particular resource/service. Suppose that $m$ pieces of the resource are available, which can satisfy $m$ simultaneous demands, but not $m+1$ or more simultaneous demands. The random demands have durations $d_{i}$ and are represented by segments with lengths $d_{i}$; the operational interval is represented by a segment with length $L$ (Fig.6).

The risk of unsatisfied demand is estimated with the expected fraction of the time interval $(0, L)$ where more than $m$ overlapping random demands are simultaneously present. (Fig.6).

Before determining the expected time fraction of simultaneously present random demands, the following theorem will be proved. An overlapped region of order $k$ is present if, for a particular point from the time interval $0, L$, exactly $k$ random demands are simultaneously present (an overlapping of order $k$ is present). The duration ratios of the random demands are given by $\psi_{i}=d_{i} / L, i=1, \ldots, n$.

Theorem 1. The expected overlapped fraction of order $k(k=0,1, \ldots, n)$ from the time interval $(0, L)$, by $n$ overlapping events with duration fractions $\psi_{i}$, coming from a distribution with mean $\bar{\psi}$, is given by the $k+1 s t$ term of the binomial expansion $[(1-\bar{\psi})+\bar{\psi}]^{n}=\sum_{i=0}^{n}\left(\begin{array}{l}n \\ i\end{array}\right) \bar{\psi}^{i}(1-\bar{\psi})^{n-i}=1$, where $1-\bar{\psi} \quad$ is treated as a single variable $a=(1-\bar{\psi})$ and $\bar{\psi}$ is treated as another variable $b=\bar{\psi}$.

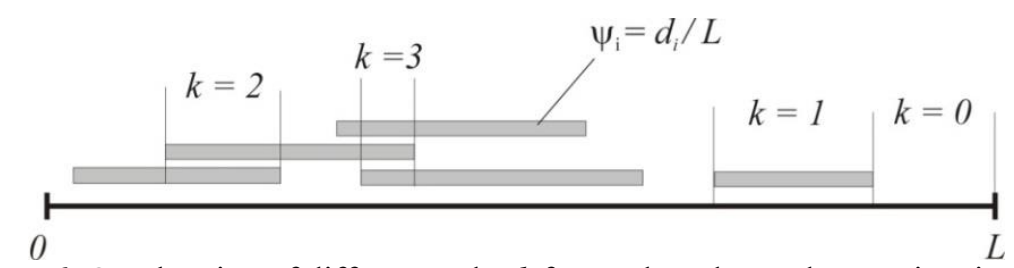

Figure 6. Overlapping of different order $k$ for random demands on a time interval.

Proof. The expected fraction of the interval $(0, L)$ covered by exactly $m$ random events can be determined from the probability that a randomly selected point on the time interval $0, L$ will sample simultaneously exactly $m$ overlapping random demands. The probability that a randomly selected point on the time interval $(0, L)$ will sample simultaneously exactly $m$ overlapping demands is equal to the probability that a fixed point from the time interval $(0, L)$ will be covered exactly $m$ times by randomly placed random demands in the time interval $(0, L)$. The probability $P\left(A_{m}\right)$ that a fixed point from the interval $(0, L)$ will be covered by exactly $m$ random demands out of $n$ random demands with duration time fractions $\psi_{1}, \psi_{2}, \ldots$, $\psi_{n}$ sampled from a distribution with mean $\bar{\psi}$ is given by

$$
P\left(A_{m}\right)=\sum_{i 1, \ldots, j m}\left(\psi_{i 1} \psi_{i 2} \ldots \psi_{i m} \prod_{\substack{k=1 \\ k \neq i 1, \ldots k \neq i m}}^{n}\left(1-\psi_{k}\right)\right)
$$

where $\sum_{i 1, \ldots, j m}$ is the sum over all distinct combinations of $m$ indices $i 1, i 2, \ldots, i m$ out of $n$ indices $1,2, \ldots, n$. The number of the combinations of $m$ out of $n$ indices is $\left(\begin{array}{l}n \\ m\end{array}\right)=\frac{n !}{m !(n-m) !}$. Equation (5) gives the sum of the probabilities of $\frac{n !}{m !(n-m) !}$ mutually exclusive events, 
covering all the cases where exactly $m$ random demands cover the selected point and the rest of the random demands $(n-m)$ do not.

Next, the expected values of the left and right hand side of equation (5) are taken:

$$
E\left[P\left(A_{m}\right)\right]=E\left\lfloor\sum_{i 1, \ldots, m}\left(\psi_{i 1} \psi_{i 2} \ldots \psi_{i m} \prod_{\substack{k=1 \\ k \neq i 1, \ldots k \neq i m}}^{n}\left(1-\psi_{k}\right)\right\rfloor\right.
$$

Because the time fractions $\psi_{k}$ of the overlapping random demands are statistically independent random variables, according to a well-known result in statistics, the expectation of a product of statistically independent random variables is equal to the product of the expectations of the random variables. Consequently, equation (6) becomes:

$$
\begin{aligned}
E\left[P\left(A_{m}\right)\right]= & \alpha=\sum_{i 1, i 2, \ldots, m} E\left(\psi_{i 1} \psi_{i 2} \ldots \psi_{i m} \times \prod_{\substack{k=1 \\
k \neq i 1, \ldots ; k \neq i m}}^{n}\left(1-\psi_{k}\right)\right)= \\
& =\sum_{i 1, i 2, \ldots, m} E\left[\psi_{i 1}\right] E\left[\psi_{i 2}\right] \ldots E\left[\psi_{i m}\right] \times \prod_{\substack{k=1 \\
k \neq i 1 ; \ldots ; k \neq i m}}^{n}\left(1-E\left[\psi_{k}\right]\right)=\left(\begin{array}{l}
n \\
m
\end{array}\right) \bar{\psi}^{m}(1-\bar{\psi})^{n=m}
\end{aligned}
$$

where $\alpha$ is the expected overlapped time fraction of order $m$ and $\bar{\psi}$ is the mean of the distribution from which the demand time fractions are sampled.

As a result, the expected overlapped time fractions of different order are given by the separate terms of the binomial expansion of

$$
[(1-\bar{\psi})+\bar{\psi}]^{n}=1
$$

where $(1-\bar{\psi})$ is treated as a single variable $a=(1-\bar{\psi})$ and $\bar{\psi}$ is treated as another variable $b=\bar{\psi}$.

$$
[(1-\bar{\psi})+\bar{\psi}]^{n}=\sum_{i=0}^{n}\left(\begin{array}{l}
n \\
i
\end{array}\right) \bar{\psi}^{i}(1-\bar{\psi})^{n-i}=1
$$

Thus, the expected time fraction $\alpha$ from the interval $(0, L)$, covered by exactly $m$ random demands, is given by

$$
\alpha=\left(\begin{array}{c}
n \\
m
\end{array}\right) \bar{\psi}^{m}(1-\bar{\psi})^{n-m}
$$

The expected covered fraction of order $m$, from the time interval $(0, L)$, depends only on the mean of the distribution of the demand times and does not depend on the type of the distribution of the demand times or the variance of the demand times.

Now consider a case where $n$ random demands come from a distribution with mean $\bar{d}$. The demand time fractions of the separate demands are $\psi_{i}=d_{i} / L, i=1, \ldots, n$. The mean of the demand time fractions $\psi_{i}$ is given by $\bar{\psi}=\bar{d} / L$. Suppose that a single source can satisfy only a single random demand at a time. The next result then holds.

For $n$ random demands and $m$ sources servicing the demands, the expected time fraction during which $m+1$ or more demands are simultaneously present on the time interval is given by the expression

$$
\alpha_{\geq m+1}=1-\sum_{i=0}^{m}\left(\begin{array}{l}
n \\
i
\end{array}\right) \bar{\psi}^{i}(1-\bar{\psi})^{n-i}
$$

where $\bar{\psi}$ is the mean of the distribution of the duration fractions of the demand times. The expected time fraction of unsatisfied demand does not depend on the distribution of the demands or their variance. 
This result follows directly from the previous theorem and its proof will be omitted.

Now consider a case where $n$ consumers demand a particular resource, during an operating period with length $L$. Suppose that the demands time fractions from each consumer come from a separate distribution with mean $\overline{\psi_{i}}(i=1, \ldots, n)$.

Theorem 2. The expected overlapped fractions of order $0,1,2, \ldots n$, for $n$ overlapping events on a time interval are given by the terms of the expansion $\prod_{i=1}^{n}\left[\left(1-\overline{\psi_{i}}\right)+\bar{\psi}_{i}\right]=1$ where $\left(1-\bar{\psi}_{i}\right)$ is treated as a single variable $a_{i}=\left(1-\overline{\psi_{i}}\right)$ and $\bar{\psi}_{i}$ is treated as another variable $b_{i}=\bar{\psi}_{i}$.

This theorem can be proved easily by induction. If all demands time fractions come from the same distribution with mean $\bar{\psi}$, the binomial expansion (8) is obtained.

These theorems and the conclusion that the expected fraction of unsatisfied demand does not depend on the distribution of the demand times have been verified by Monte Carlo simulations involving direct measurement and accumulation of the overlapping regions.

If the time fraction of the durations of the random demands is constant: $\psi=d / L$, the expected (average) fraction of time during which exactly $m$ random demands are simultaneously present $(m=0,1, \ldots, n)$ is given by the terms $\frac{n(n-1) \ldots(n-m+1)}{1 \times 2 \times \ldots \times m}(1-\psi)^{n-m} \psi^{m}$ , which come from the binomial expansion of the expression $[(1-\psi)+\psi]^{n}$.

Thus, $(1-\psi)^{n}$ is the expected fraction of time during which no random demand is present; $n(1-\psi)^{n-1} \psi^{1}$ is the expected fraction of time during which exactly one random demand is present; $\frac{n(n-1)}{1 \times 2}(1-\psi)^{n-2} \psi^{2}$ is the expected fraction of time during which exactly two random demands are simultaneously present; $\ldots ; \psi^{n}$ is the expected fraction of time during which exactly $n$ random demands are simultaneously present. The sum of all expected fractions of time is equal to one $\left([(1-\psi)+\psi]^{n}=1\right)$.

Because the sum of all expected fractions is equal to one, the total expected fraction of time during which there is unsatisfied demand $(m+1$ or more random demands are simultaneously present) is given by

$$
\alpha_{\geq m+1}=1-\left((1-\psi)^{n}+n(1-\psi)^{n-1} \psi^{1}+\frac{n(n-1)}{1 \times 2}(1-\psi)^{n-2} \psi^{2}+\ldots+\frac{n(n-1) \ldots(n-m)}{1 \times 2 \times \ldots \times m}(1-\psi)^{n-m} \psi^{m}\right)
$$

Now consider the important case of $n$ consumers, each placing a random demand with probability $\gamma$, during the interval $(0, L)$. The time fractions of the random demands come from a distribution with mean $\bar{\psi}$. The next theorem then holds.

Theorem 3. The expected time fractions of different order, for $n$ consumers each placing a random demand with probability $\gamma$, are given by the terms of the binomial expansion of $[(1-\gamma \bar{\psi})+\gamma \bar{\psi}]^{n}=1$, where $1-\gamma \bar{\psi}$ is treated as a single variable $a=(1-\gamma \bar{\psi})$ and $\gamma \bar{\psi}$ is treated as another variable $b=\gamma \bar{\psi}$.

Proof. Placing a random demand with probability $0 \leq \gamma \leq 1$ is equivalent to sampling the demand time fraction from a distribution mixture defined as follows: The time fractions of the random demands are sampled with probability $\gamma$ from the distribution with mean $\bar{\psi}$ and with probability $1-\gamma$ from the constant zero. Indeed, sampling zero demand time with probability $1-\gamma$ means a random demand with zero time fraction $(\psi=0)$. A zero demand time fraction cannot possibly contribute to the expected overlapped fraction of any order and is equivalent to not having a random demand, with probability $1-\gamma$. 
The mean of the as-defined distribution mixture is $\mu=\gamma \times \bar{\psi}+(1-\gamma) \times 0=\gamma \times \bar{\psi}$. According to Theorem 1, the expected overlapped time fraction of a particular order is the same irrespective of whether random demands with constant demand fraction equal to $\gamma \times \bar{\psi}$ are present on the time interval $(0, L)$ or the demand fractions come from the as-defined distribution mixture with mean $\mu=\gamma \times \bar{\psi}$. Because for demands with constant time fractions $\mu=\gamma \bar{\psi}$, the expected overlapped time fraction of any particular order is given by the terms of the binomial expansion $[(1-\gamma \bar{\psi})+\gamma \bar{\psi}]^{n}=1$, the theorem has been proven.

The total expected fraction of time during which there is unsatisfied demand $(m+1$ or more random demands are simultaneously present) is then given by

$$
\alpha_{\geq m+1}=1-\sum_{i=0}^{m}\left(\begin{array}{l}
n \\
i
\end{array}\right)(\gamma \bar{\psi})^{i}(1-\gamma \bar{\psi})^{n-i}
$$

The theorem has been verified by Monte Carlo simulations.

From equation (12) it can be seen that for a fixed number of consumers initiating demands with certain probability $\gamma$, reducing the mean $\bar{\psi}$ of the demand times by a certain factor $k>1$ and reducing the probability of initiating the random demands $\gamma$ by the same factor $k>1$ has the same effect on the degree of stochastic separation between the random demands. Alternatively, the degree of stochastic separation does not change if an increase of the mean $\bar{\psi}$ of the demand times by a factor $k>1$ has been compensated by a decrease by the same factor of the probability $\gamma$ of initiating a random demand: $(k \bar{\psi}) \times(\gamma / k)=\bar{\psi} \gamma$.

Consider the following example. For an operational time interval $L=58$ hours, a single source $(m=1)$ is available for servicing the demands from $n=150$ consumers, whose demand durations follow a uniform distribution within the range $(10 \mathrm{~min}, 40 \mathrm{~min})$ (with mean 25 minutes; and mean demand time fraction $\bar{\psi}=25 /(58 \times 60))$. The probability with which each consumer initiates a random demand is $\gamma=0.6$. For $m=1$ sources servicing the random demands, equation (12) results in $\alpha_{\geq 2}=1-(1-\gamma \bar{\psi})^{n}-n(\gamma \bar{\psi}) \times(1-\gamma \bar{\psi})^{n-1}$ which, after the substitution of the numerical values, gives $\alpha_{\geq 2}=0.137$ for the expected fraction of unsatisfied demand. This value has been confirmed by the Monte Carlo simulation result: $\alpha_{\geq 2}=0.137$.

Next, the demand durations were distributed uniformly in the following intervals: $25 \pm 0$ $25 \pm 5 \mathrm{~min}, 25 \pm 10 \mathrm{~min}, 25 \pm 15 \mathrm{~min}, 25 \pm 20$ and $25 \pm 25$, all of which had the same mean of $25 \mathrm{~min}$. The simulations for each of the intervals resulted in the same expected fraction of unsatisfied demand equal to 0.137 . These results confirm the earlier conclusion that the expected fraction of unsatisfied demand is independent of the variance of the demand times.

Consider finally the case where each of the $n$ consumers initiates a demand with probability $\gamma_{i}$ and the demand time fractions characterising the consumer come from a separate distribution with mean $\bar{\psi}_{i}(i=1, \ldots, n)$. Theorem 2 , can then be naturally generalised to the next theorem.

Theorem 4. The expected overlapped fractions of order $0,1,2, \ldots n$, for $n$ overlapping events on a time interval are given by the separate terms of the expansion $\prod_{i=1}^{n}\left[\left(1-\gamma_{i} \bar{\psi}_{i}\right)+\gamma_{i} \bar{\psi}_{i}\right]=1$ where $\left(1-\gamma_{i} \bar{\psi}_{i}\right)$ is treated as a single variable $a_{i}=\left(1-\gamma_{i} \bar{\psi}_{i}\right)$ and $\gamma_{i} \bar{\psi}_{i}$ is treated as another variable $b_{i}=\gamma_{i} \bar{\psi}_{i}$. 


\section{Stochastic separation based on the expected fraction of unsatisfied demand}

\subsection{Fixed number of random demands on a time interval}

Consider a finite time interval during which a number of consumers place a single demand for a particular service independently and randomly, during a time interval $0, L$. The duration times come from a distribution with mean $d$. The mean demand time fraction is therefore $\bar{\psi}=d / L$. Unsatisfied demand occurs if a random demand arrives while all $m$ available sources are engaged in servicing random demands. Suppose that the maximum tolerable expected fraction of unsatisfied demand is $\alpha$.

Solving equation (13) with respect to $n$ then yields the maximum number of random demands that can be serviced:

$$
\alpha=1-\sum_{i=0}^{m}\left(\begin{array}{l}
n \\
i
\end{array}\right) \bar{\psi}^{i}(1-\bar{\psi})^{n-i}
$$

This equation can be solved by a repeated bisection.

Figure 7 gives the expected fraction of unsatisfied demand as a function of the number $n$ of random demands. The figure corresponds to a demand time fraction $\bar{\psi}=d / L=0.15$ of an individual demand. The specified maximum acceptable expected fraction of unsatisfied demand was $\alpha=10 \%$ and the number of available sources was $m=3$.

By using repeated bisection, it was determined that at most 12 users can be serviced by the sources without exceeding the specified level $\alpha=10 \%$ of expected fraction of unsatisfied demand.

At another specified level of the expected fraction of unsatisfied demand (for example, $\alpha=20 \%$ ), the maximum number $n$ of consumers that can be serviced can be determined directly from the curve. As can be verified from the plots in Fig.7, increasing the number of sources $m$ increases significantly the number of consumers that can be serviced without exceeding the tolerable risk of unsatisfied demand.

Suppose that the number of consumers $n$ (each placing a single random demand on the time interval) and the maximum tolerable expected fraction of unsatisfied demand $\alpha$ have been specified. Solving equation (13) with respect to $m$ now yields the number of sources required to service the $n$ consumers such that the expected fraction of unsatisfied demand does not exceed $\alpha$. The solutions complying with these requirements provide a stochastic separation of random events based on the expected overlapped fraction. The stochastic separation based on the expected overlapping fraction, guarantees that the expected degree of overlapping will be equal to or below a specified tolerable level.

Finding the minimum number of sources which guarantees a degree of stochastic separation based on the expected overlapped fraction is critical in striking the right balance between the risk of unsatisfied demand and costs. If the sources are medical personnel, repairmen or extra equipment, increasing the number of sources increases the degree of stochastic separation but also increases salary costs or investment, which undermines profit. Too few sources means increased risk of unsatisfied demand, risk of fatalities, damage to health, dissatisfied customers, etc. Again, equation (13) can be solved by a repeated bisection with respect to $m$, by keeping the number of consumers $n$ and the demand time fraction $\bar{\psi}=d / L$ constant. Figure 8 gives the expected time fraction of unsatisfied demand as a function of the number of sources, for different demand time fractions $\bar{\psi}=d / L=0.1,0.2,0.3,0.4,0.5,0.6$. The number of consumers $n$ is fixed $(n=20)$. 


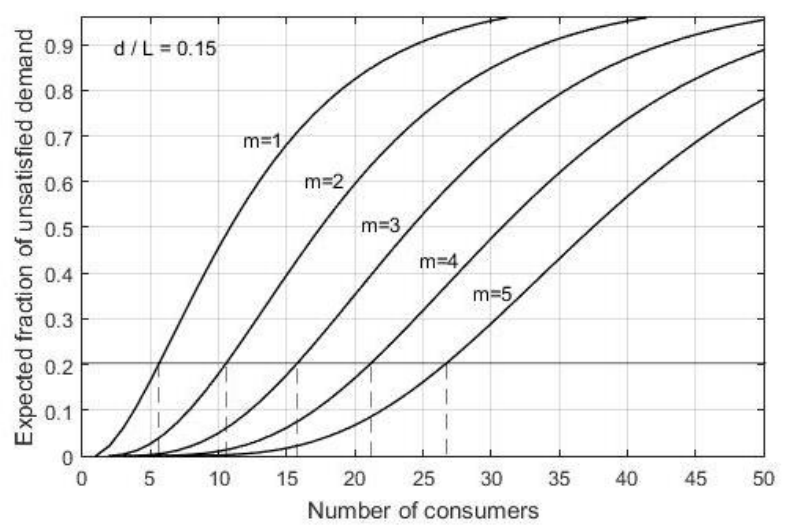

Figure 7. Expected fraction of unsatisfied random demand as a function of the number of consumers $n$, for different number of sources $m$ servicing the demands.

The maximum acceptable level of the expected fraction of unsatisfied demand has been specified to be $\alpha=10 \%$; the number of consumers have been specified to be and $n=20$, each characterised by a demand time fraction $\bar{\psi}=d / L=0.2$. For these input data, the repeated bisection algorithm determined that the minimum number of sources must be 6 , in order to achieve the $10 \%$ expected fraction of unsatisfied demand which is the specified degree of stochastic separation between the random events.

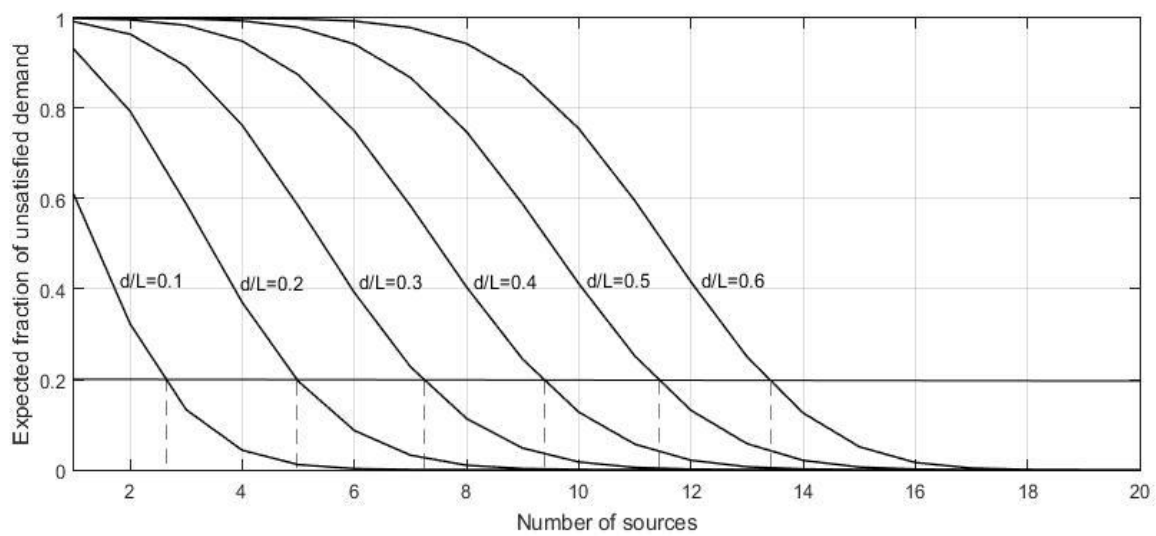

Figure 8. The expected fraction of unsatisfied demand as a function of the number of sources, at different demand time fractions $\psi=d / L$.

At a specified level of the expected fraction of unsatisfied demand (for example, $\alpha=20 \%$ ), the minimum number of sources that can guarantee a degree of stochastic separation (expected fraction of unsatisfied demand) of $\alpha=20 \%$ or below can also be determined directly from the curves. As can be verified from the graphs, increasing the mean demand fraction $\bar{\psi}=d / L$ increases significantly the number of sources needed to service the random demands so that a degree of stochastic separation below the maximum tolerable level $\alpha$ is guaranteed.

\subsection{Random demands following a Poisson process on a time interval}

Suppose now that the random demands follow a homogeneous Poisson process with density $\lambda$, on a time interval with length $L$. The number of available sources is $m$. The durations of the random demands follow a particular distribution with mean $\bar{\psi}$. Unsatisfied demand is present only if the number of random demands is greater than the number of available sources $m$. The expected fraction of unsatisfied demand $\alpha$ on the time interval $(0, L)$ is equal 
to the probability that a randomly selected point on the time interval $0, L$ will sample simultaneously exactly $m+1$ or more overlapping random demands. The probability that a randomly selected point on the time interval $(0, L)$ will sample $m+1$ or more overlapping demands is equal to the probability that a fixed point from the time interval $(0, L)$ will be covered $m+1$ or more times by randomly placed random demands in the time interval $(0, L)$.

A selected point in the time interval $(0, L)$ can be covered $m+1$ or more times in the following mutually exclusive ways: there are exactly $m+1$ random demands in the interval $(0, L)$ and the selected point has been covered by all of them; there are exactly $m+2$ random demands in the time interval $(0, L)$ and the selected point is covered $m+1$ or more times, there are exactly $m+3$ random demands in the time interval $(0, L)$ and the selected point is covered $m+1$ or more times and so on. The probability $\alpha$ that the selected point will be covered $m+1$ or more times can then be determined as a sum of the probabilities of the following mutually exclusive events: (i) exactly $m+1$ demands are present in the time interval (event $A_{m+1}$ characterised by a probability $\left.P\left(A_{m+1}\right)\right)$ and the selected point is covered by $m+1$ or more random demands (event $B$ characterised by a conditional probability $P(B \mid m+1)$; (ii) exactly $m+2$ demands are present in the time interval (event $A_{m+2}$ characterized by a probability $P\left(A_{m+2}\right)$ ) and the selected point is covered by $m+1$ or more random events (event $B$ characterised by a conditional probability $P(B \mid m+2)$ and so on. For the probability $\alpha$ of covering the selected point, the total probability theorem yields the expression:

$$
\alpha=P\left(A_{m+1}\right) \times P(B \mid m+1)+P\left(A_{m+2}\right) \times P(B \mid m+2)+\ldots
$$

For random demands following a Homogeneous Poisson process with density $\lambda$, on a time interval with length $L$, the probability that there will be exactly $m+1$ random demands on the time interval $0, L$ is obtained from $P\left(A_{m+1}\right)=\frac{e^{-\lambda L}}{(m+1) !}(\lambda L)^{m+1}$; the probability that there will be exactly $m+2$ random demands on the time interval $0, L$ is given by $P\left(A_{m+2}\right)=\frac{e^{-\lambda L}}{(m+2) !}(\lambda L)^{m+2}$ and so on. According to Section 4, the probability that the selected point will be covered with order $m+1$ or higher, given that there are $n \geq m+1$ random demands on the time interval, is given by $P(B \mid n)=1-\sum_{i=0}^{m}\left(\begin{array}{l}n \\ i\end{array}\right) \bar{\psi}^{i}(1-\bar{\psi})^{n-i}$. For $n \leq m$, the probability that the selected point will be covered with order $m+1$ or higher is zero.

The substitution in equation (14) yields

$$
\alpha=\sum_{n=m+1}^{\infty} \frac{e^{-\lambda L}}{n !}(\lambda L)^{n}\left[1-\sum_{i=0}^{m}\left(\begin{array}{l}
n \\
i
\end{array}\right) \bar{\psi}^{i}(1-\bar{\psi})^{n-i}\right]
$$

for the probability that the selected point will be covered: This is also the fraction $\alpha$ of unsatisfied demand.

For practical purpose, the computation of the sum (15) can be truncated with negligible error at $n=N$, which is, for example, ten times larger than the average number $\lambda L$ of demands in the time interval $0, L(N=10 \times(\lambda L))$. Equation (15) then becomes

$$
\alpha=\sum_{n=m+1}^{N} \frac{e^{-\lambda L}}{n !}(\lambda L)^{n}\left[1-\sum_{i=0}^{m}\left(\begin{array}{l}
n \\
i
\end{array}\right) \bar{\psi}^{i}(1-\bar{\psi})^{n-i}\right]
$$

Suppose that a single source is servicing random demands following a homogeneous Poisson process on a time interval. In this case, $m=1$ and equation (16) becomes 


$$
\alpha=\sum_{n=2}^{N} \frac{e^{-\lambda L}}{n !}(\lambda L)^{n}\left[1-(1-\bar{\psi})^{n}-n(1-\bar{\psi})^{n-1} \bar{\psi}\right]
$$

Thus, for $L=68$ hours, $\lambda=7$ hour $^{-1}$, average duration of a single demand $d=15 \mathrm{~min}$ and a single source ( $m=1$ ), equation (17) yields $\alpha=0.52$. This value has been confirmed by the Monte Carlo simulation.

For two sources servicing random demands following a homogeneous Poisson process on a time interval ( $m=2)$, equation (16) becomes

$$
\alpha=\sum_{n=3}^{N} \frac{e^{-\lambda L}}{n !}(\lambda L)^{n}\left[1-(1-\bar{\psi})^{n}-n(1-\bar{\psi})^{n-1} \bar{\psi}-\frac{n(n-1)}{2}(1-\bar{\psi})^{n-2} \bar{\psi}^{2}\right]
$$

For the same input data set as in the previous example and two sources $(m=2)$ servicing the random demands, equation (18) yields $\alpha=0.256$, which has also been confirmed by the Monte Carlo simulation.

For three sources servicing random demands following a homogeneous Poisson process on a time interval $(m=3)$, equation (16) becomes

$$
\begin{aligned}
\alpha=\sum_{n=4}^{N} \frac{e^{-\lambda L}}{n !}(\lambda L)^{n} \times & \\
& {\left[1-(1-\bar{\psi})^{n}-n(1-\bar{\psi})^{n-1} \bar{\psi}-\frac{n(n-1)}{2 !}(1-\bar{\psi})^{n-2} \bar{\psi}^{2}-\frac{n(n-1)(n-2)}{3 !}(1-\bar{\psi})^{n-3} \bar{\psi}^{3}\right] }
\end{aligned}
$$

For the same input data set as in the previous example and three sources $(m=3)$ servicing the demands, equation (19) yields $\alpha=0.1$, which has also been confirmed by the Monte Carlo simulation.

As can be verified, in the case of random demands following a homogeneous Poisson process, including additional sources significantly reduces the expected fraction of unsatisfied demand.

Using the closed-form expressions (16) and (12) is significantly more efficient compared to estimating the expected fraction of unsatisfied demand by a direct Monte Carlo simulation. The closed-form expressions permit their embedding in ultra-fast optimisation loops thereby reducing dramatically the computation time. Furthermore, the closed-form expressions also permit important inferences to be made about the properties of a system consisting of consumers placing random demands on a time interval.

The presented techniques related to reducing the risk of overlapping of risk-critical events by stochastic separation, could become an important extension of the method of separation for reliability improvement and risk reduction, recently introduced in [25].

\section{Modelling the dependence of the probability of overlapping of random events on the events durations}

Suppose that $n$ events with durations $d_{1}, \ldots, d_{n}$, appear randomly on a time interval $0, L$ ( $\left.d_{1}+d_{2}+\ldots+d_{n}<L\right)$ such that no overlapping of random events is present (Fig.9a). The events start times $s_{1}, s_{2}, \ldots, s_{n}$ are uniformly distributed along the interval $(0, L)$. The configuration in Fig.9a will be referred to as $X$-configuration. Note that the duration of the last event cannot possibly contribute to overlapping on the time interval $(0, L)$ and therefore can be ignored. The first $n-1$ duration intervals $d_{1}, \ldots, d_{n-1}$ can then be 'cut out' of the time interval $(0, L)$ and the remaining parts of the time interval can be 'brought together' to form a shorter length $L-\left(d_{1}+d_{2}+\ldots+d_{n-1}\right)$ (Fig.9b). As a result of this operation, the points $s_{1}, s_{2}, \ldots, s_{n}$ marking the start of the random events for the $X$-configuration transform into a 
unique $Y$-configuration where the points $s_{1}^{\prime}, s_{2}^{\prime}, \ldots, s_{n}^{\prime}$ are uniformly distributed along the length $L-\left(d_{1}+d_{2}+\ldots+d_{n-1}\right)$. As a result, to each $X$-configuration corresponds exactly one $Y$-configuration. Thus, for the set of all possible $X$-configurations and the set of all possible $Y$-configurations, the relationship $X \subseteq Y$ holds.
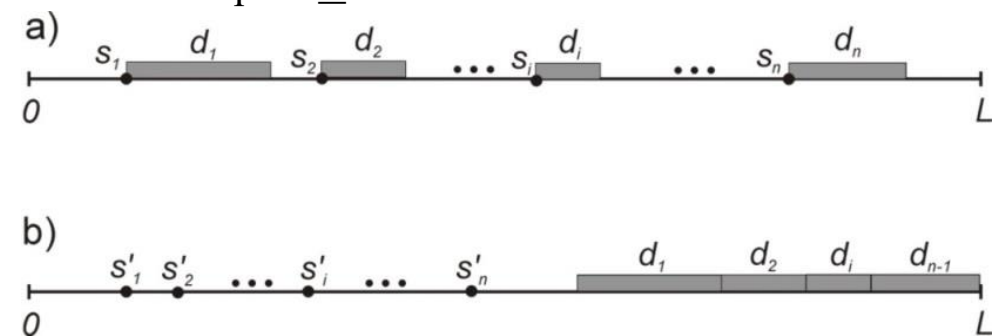

Figure 9. Scheduling random events in a finite time interval $(0, L)$ by guaranteeing a full time separation

Now suppose that a set of $n$ points $s_{1}^{\prime}, s_{2}^{\prime}, \ldots, s_{n}^{\prime}$ are uniformly distributed along the length $L$. If all $n$ randomly generated points fall within the length $L-\left(d_{1}+d_{2}+\ldots+d_{n-1}\right)$ a $Y$ configuration is present. By inserting the corresponding demand intervals $d_{i}(i=1, \ldots, n-1)$ after each start time $s_{i}$, an $X$-configuration can be obtained with random non-overlapping events along the interval $0, L$.

Thus, from each $Y$-configuration, a unique $X$-configuration is obtained by placing after each randomly generated points $s_{i}^{\prime}$ a duration interval with length $d_{1}, \ldots, d_{n-1}$ The start times in the $X$-configuration (Fig.9a) are therefore: $s_{1}=s_{1}, s_{2}=s_{2}^{\prime}+d_{1}, s_{3}=s_{3}^{\prime}{ }_{3}+d_{1}+d_{2}, \ldots$, $s_{n}=s_{n}^{\prime}+d_{1}+d_{2}+\ldots+d_{n-1}$. In the obtained $X$-configuration all random events are fully separated. As a result, to each $Y$-configuration corresponds exactly one $X$-configuration. Thus, for the set of all possible $Y$-configurations and the set of all possible $X$-configurations, the relationship $Y \subseteq X$ holds.

Since for the sets of the $X$-configurations and $Y$-configurations $Y \subseteq X$ and $X \subseteq Y$ simultaneously hold, there exists a one-to-one correspondence between the $X$-configurations, characterised by randomly located non-overlapping random events and the $Y$-configurations, characterised by randomly located random points on the smaller time interval $L-\left(d_{1}+d_{2}+\ldots+d_{n-1}\right)$. Because of the one-to-one correspondence between $X$-configurations and $Y$-configurations, the probability of an $X$-configuration can be measured by the probability of a $Y$-configuration.

Suppose that the start times of the events are uniformly distributed along the length of the time interval $(0, L)$. Let $A_{1}, A_{2}, \ldots, A_{n}$ denote the events 'the last event has a duration $d_{1}, d_{2}, \ldots$, $d_{n}$, correspondingly. The probability of the event $B$ that there will be no overlapping of random events can be determined by the following probabilistic argument.

Initially, the conditional probability $P\left(B \mid A_{n}\right)$ is determined - the probability that there will be no overlapping of random events, given that the last event has a duration $d_{n}$. Because each random event has an equal chance to be the last event, the probabilities $p\left(A_{i}\right)$ of the events $A_{i}$ are all equal to $1 / n\left(p\left(A_{i}\right)=1 / n, i=1,2, \ldots, n\right)$.

The probability of an $Y$-configuration that all $n$ uniformly distributed random points $s_{1}^{\prime}, s_{2}^{\prime}, \ldots, s_{n}^{\prime}$ along the interval $(0, L)$ will fall in the interval $L-\left(d_{1}+d_{2}+\ldots+d_{n-1}\right)$ is given by $\left(\frac{L-\left(d_{1}+\ldots+d_{n-1}\right)}{L}\right)^{n}$. This is also the conditional probability of an $X$-configuration (no 
overlapping events) given that the last event has a duration $d_{n}$.

$$
P\left(B \mid A_{n}\right)=\left(1-\frac{\left(d_{1}+\ldots+d_{n-1}\right)}{L}\right)^{n}
$$

However, the absence of overlapping (event $B$ ) can occur in $n$ different ways. The absence of overlapping can occur given that the last random event has a duration $d_{n}$, given that the last random event has a duration $d_{n-1}, \ldots$, and so on. The probabilities $P\left(B \mid A_{i}\right), i=1, \ldots, n-1$, are determined in a similar fashion. According to the total probability theorem,

$$
P(B)=P\left(B \mid A_{1}\right) P\left(A_{1}\right)+\ldots+P\left(B \mid A_{n}\right) P\left(A_{n}\right)
$$

As a result, the expression

$$
P(B)=\frac{1}{n}\left[\left(1-\frac{d_{2}+d_{3}+\ldots+d_{n}}{L}\right)^{n}+\left(1-\frac{d_{1}+d_{3}+\ldots+d_{n}}{L}\right)^{n}+\ldots+\left(1-\frac{d_{1}+d_{2}+\ldots+d_{n-1}}{L}\right)^{n}\right]
$$

is obtained for the probability of a full separation (no overlapping) of random events with durations $d_{1}, \ldots, d_{n}$. Equation (22) can also be presented as

$$
P(B)=\frac{1}{n}\left[\left(1-\frac{D-d_{1}}{L}\right)^{n}+\left(1-\frac{D-d_{2}}{L}\right)^{n}+\ldots+\left(1-\frac{D-d_{n}}{L}\right)^{n}\right]
$$

where $D=d_{1}+d_{2}+\ldots+d_{n}$. Equation (22) has been confirmed by the results from Monte Carlo simulations. Thus, for four consumers demanding a particular resource for $d_{1}=5 \mathrm{~min}$, $d_{2}=10 \mathrm{~min}, d_{3}=20 \mathrm{~min}$, and $d_{4}=35 \mathrm{~min}$ respectively, during a time interval of 10 hours, the probability of no overlapping calculated from equation (22) is 0.7 . This probability has been confirmed by the probability of 0.7 estimated from the simulation. (The details of the simulation algorithm have been omitted). For a given set of events with duration times $d_{1}, \ldots$, $d_{n}$ two types of questions related to the stochastic separation can be posed:

- The smallest length $L$ of the time interval which provides a stochastic separation with a specified probability $P(B)$.

- The maximum number of events $n$ with specified durations which still provides a stochastic separation of specified probability $P(B)$.

Both questions can be answered by solving the non-linear equation (23) with respect to $L$ or with respect to $n$.

Suppose that four consumers are demanding a particular resource for $d_{1}=5 \mathrm{~min}$, $d_{2}=10 \mathrm{~min}, d_{3}=20 \mathrm{~min}$, and $d_{4}=35 \mathrm{~min}$ respectively. If the probability of no overlapping has been specified to be 0.8 , the nonlinear equation

$$
f(L) \equiv \frac{1}{4}\left[\left(1-\frac{D-d_{1}}{L}\right)^{4}+\left(1-\frac{D-d_{2}}{L}\right)^{4}+\left(1-\frac{D-d_{3}}{L}\right)^{4}+\left(1-\frac{D-d_{4}}{L}\right)^{4}\right]-0.8=0
$$

can be solved with respect to $L$ by a repeated bisection in the interval $L_{\min }(100 \mathrm{~min}) \leq L \leq L_{\max }(2000 \mathrm{~min})$ because at the ends of this interval $f(L)$ has different signs $\left(f\left(L_{\min }\right)<0\right.$ and $\left.f\left(L_{\max }\right)>0\right)$. The solution of equation (24), obtained by using a standard repeated bisection algorithm, is $L=16$ hours. Thus, in order to provide the required separation probability of 0.8 , the length of the time interval needs to be 16 hours.

Suppose that the durations $x_{1}, x_{2}, \ldots, x_{n-1}, x_{n}$ of the fists, second,...,nth random event are realisations of a random variable $X$ following a statistical distribution with mean $\mu$ and 
standard deviation $\sigma$. According to equation (23), the probability that the random events will be separated (will not overlap) are given by

$$
P(B)=\frac{1}{n}\left[\left(1-\frac{D-x_{1}}{L}\right)^{n}+\left(1-\frac{D-x_{2}}{L}\right)^{n}+\ldots+\left(1-\frac{D-x_{n}}{L}\right)^{n}\right]
$$

where $D=\sum_{i=1}^{n} x_{i}$. Even for a relatively small number of events, the sum of the event durations $\sum_{i=1}^{n} x_{i}-x_{1}, \sum_{i=1}^{n} x_{i}-x_{1}, \ldots, \sum_{i=1}^{n} x_{i}-x_{n}$ in equation (25) can be approximated reasonably well with $(n-1) \mu$ and, as a result, the probability $P(B)$ of a full separation (non-overlapping) of random events is given by

$$
P(B) \approx\left(1-\frac{(n-1) \mu}{L}\right)^{n}
$$

This probability is practically insensitive to the variance of the random variable $X$ standing for the durations of the demand times. The probability $P(B)$ depends on the expected value $\mu=E(X)$ of the demand times. The probability of event separation is practically insensitive to the variance (standard deviation $\sigma$ ) of the demand times $X$.

This conclusion has been verified by numerous computer simulations where the probability of overlapping has been plotted as a function of the variance of the duration times.

The practical insensitivity of the probability of full event separation to the variance of the duration times, for a very small number of random events, can be understood from analysing the expression for the probability of no overlapping of two random events. From equation (22), this probability is:

$$
P(B)=\frac{1}{2}\left[\left(1-x_{2} / L\right)^{2}+\left(1-x_{1} / L\right)^{2}\right]
$$

where $x_{1}$ is the random variable representing the duration time of the first event and $x_{2}$ is the random variable representing the duration times of the second random event.

Expanding expression (27) results in

$$
P(B)=0.5 \times\left(1-2 x_{2} / L+x_{2}^{2} / L^{2}+1-2 x_{1} / L+x_{1}^{2} / L^{2}\right)
$$

Taking expectations from both sides of equation (28) results in

$$
E[P(B)]=0.5 \times\left(1-2 E\left(x_{2}\right) / L+E\left(x_{2}^{2} / L\right)+1-2 E\left(x_{2}\right) / L+E\left(x_{1}^{2} / L^{2}\right)\right)
$$

In equation (29), $E\left(x_{1}\right)=E\left(x_{2}\right)=\bar{x}$, where $\bar{x}$ is the mean of the duration times of the random events.

It is known that if $X$ is a random variable with finite mean and $y$ is a convex function ( $\lambda y(a)+(1-\lambda) y(b) \geq y(\lambda a+(1-\lambda) b), 0 \leq \lambda \leq 1 ; a, b \in \mathbf{R})$ then the Jensen's inequality [26] holds:

$$
E[y(x)] \geq y(E[x])
$$

The function $y=(x / L)^{n}$ is a convex function for $n \geq 1$, therefore, according to the Jensen's inequality, $E\left[(x / L)^{n}\right] \geq(\bar{x} / L)^{n}$. However, for small ratios $x_{i} / L \quad(i=1,2)$, the terms $x_{i}^{n} / L^{n}$ are very small, and the error from replacing $E\left(x_{i}^{n} / L^{n}\right)$ with $\left.\left[E\left(x_{i}\right)\right]^{n} / L^{n}\right)=\bar{x}^{n} / L^{n}$ is also very small (For the sake of brevity, the derivation details have been omitted here). Consequently,

$$
\left.E[P(B)]=P(B) \approx 1-2 \bar{x} / L+\bar{x}^{2} / L^{2}\right)=(1-\bar{x} / L)^{2}
$$

can be used for the probability of no overlapping of two random events. As a result, for small ratios $\bar{x} / L$, the probability of no overlapping of two random events is practically insensitive 
to the variance of their duration times. Equation (31) has also been verified by computer simulations. The simulation results and the theoretical analysis demonstrate that the probability of non-overlapping of random events is practically insensitive to the type of the distribution of the duration times, provided that the means of the distributions are not altered. Also, the probability of non-overlapping of random events is practically insensitive to the variance of the duration times. These results provide the valuable opportunity to work with random demand times characterised by their means only without requiring information related to the variance of the demand times.

\section{Conclusions}

- A closed-form equation has been derived and validated for the expected time fraction of simultaneous presence of random events whose times of occurrence follow a homogeneous Poisson process in a specified time interval. The equation handles any specified number of sources servicing the random events.

- A closed-form equation has been derived and validated for the expected time fraction of unsatisfied demand for a fixed number of consumers which initiate random demands with a specified probability.

- The expected time fraction of simultaneously present events, appearing randomly on a time interval, does not depend on the distribution of their duration times or on the variance of the duration times as long as the mean remains the same.

- The concepts stochastic separation of random events based on the probability of overlapping and the average overlapped fraction have been introduced for the first time. Methods for providing stochastic separation and optimal stochastic separation achieving balance between risk and cost of risk reduction have been presented.

- For a fixed number of consumers initiating demands with certain probability, reducing the mean of the demand times by a certain factor and reducing the probability of initiating the random demands by the same factor has the same effect on the degree of stochastic separation between the random demands.

- The probability of simultaneously present events, randomly appearing on a time interval, is practically insensitive to the distribution of their durations and their variance. This provides the valuable opportunity to work with duration times characterised by their means only and not requiring information related to the variance of the duration times.

- The distribution of the repair time of a failed component in a complex system, including different types of components, is given by a distribution mixture.

\section{References}

[1] Solomon H., Geometric probability, 1987, Society for industrial and applied mathematics.

[2] Stevens W.L., "Solution to a geometrical problem in probability", 1939, Ann.Eugenics, 9 pp.315-320.

[3] Shepp L.A., 1972, "Covering the circle with random arcs", Israel J.Math., 11, pp.328345.

[4] Calin O., Udriste K., 2014, Geometric modelling in probability and statistics, Springer.

[5] Huffer F.W. and Shepp L.A., 1987, "On the Probability of Covering the Circle by Random Arcs", Journal of Applied Probability, 24 (2), pp.422-429. 
[6] Coffman E.G, Mallows C.L. and Poonen B., 1994, "Parking arcs on the circle with applications to one-dimensional communication networks", The Annals of Applied Probability, 4(4), pp.1098-1111.

[7] Coffman E.G., Flatto L., Jelenkovic P., and Poonen B., 1998, "Packing Random Intervals On-Line", Algorithmica, 22, pp.448-476.

[8] Justicz J., Scheinerman E.R. and Winkler P.M., 1990, "Random intervals", Amer. Math. Monthly, 97, pp.881-889.

[9] Todinov M.T., 2016, "Evaluating the risk of unsatisfied random demand on a time interval", Artificial Intelligence Research, 5(1), pp.67-77.

[10] Khintchine, A. Y., 1969, Mathematical methods in the theory of Queueing. London: Griffin.

[11] Cooper R., 1981, Introduction to queuing theory, 2nd ed., Elsevier.

[12] Gross D., and Harris C.M., 1985, Fundamentals of queuing theory, 2nd ed., New York: Wiley.

[13] Gnedenko B.V., I.N.Kovalenko, 1989, Introduction to queuing theory, 2nd ed., Birkhauser, Berlin.

[14] Thompson W.A., 1988, Point process models with applications to safety and reliability, Chapman \& Hall.

[15] Allen A.O., 1990, Probability, Statistics and Queuing theory with computer science applications, 2nd ed., Academic press Inc.

[16] Kalashnikov V., 1994, Mathematical methods in queuing theory, SpringerScience+Business Media.

[17] Giambene G., 2005, Queuing theory and telecommunications, Networks and applications, Springer.

[18] Daigle J.N., 2005, Queueing theory with applications to packet telecommunication, Springer.

[19] Bhat U.N., 2008, An introduction to queuing theory: modelling and analysis in applications, Birkhauser Boston.

[20] Weiss N.A., 2006, A course in probability, Pearson/Addison Wesley.

[21] Stroock D.W., 2011, Probability theory: An analytical view, 2nd ed., Cambridge University press.

[22] Venkatesh S.S., 2013, The theory of probability: explorations and applications, Cambridge University Press.

[23] Todinov M.T., 2002, "Distribution mixtures from sampling inhomogeneous microstructures: variance and probability bounds of the properties", Nuclear Engineering and Design, 214, pp.195-204.

[24] Everitt, B.S., Hand, D.J., 1981, Finite Mixture Distributions. Chapman and Hall, London.

[25] Todinov M.T., 2015, "Reducing risk through segmentation, permutations, time and space exposure, inverse states, and separation", International Journal of Risk and Contingency Management, 4(3), pp.1-21.

[26] Rosenthal J.S., 2006, A first look at rigorous probability theory, 2nd ed., World Scientific Publishing Co. Pte. Ltd. 